Ciência Florestal, Santa Maria, v. 25, n. 1, p. 145-152, jan.-mar., 2015

ISSN 0103-9954

\title{
AVALIAÇÃO DA SUPERFÍCIE DA MADEIRA DE Eucalyptus grandis Hill ex Maiden TRATADA TERMICAMENTE
}

\section{SURFACE EVALUATION OF THE HEAT TREATED WOOD OF Eucalyptus grandis Hill ex Maiden}

\author{
Gilmara Pires de Moura Palermo ${ }^{1}$ João Vicente de Figueiredo Latorraca ${ }^{2}$ \\ Alexandre Monteiro de Carvalho ${ }^{3}$ Rosilei Aparecida Garcia ${ }^{4}$
}

\begin{abstract}
RESUMO
O objetivo deste estudo foi estudar a qualidade da superfície usinada da madeira de Eucalyptus grandis, submetida ao processo de tratamento térmico. As operações de usinagens realizadas nas tábuas obtidas a partir do processamento de mecânico de seis árvores foram: desempeno, desengrosso, rasgo na furadeira horizontal, furação, furação para dobradiça e lixamento. Foram selecionadas 208 tábuas de 125 x 25 x 500 $\mathrm{mm}$, sendo 104 não tratadas e 104 tratadas termicamente a uma temperatura final de $190^{\circ} \mathrm{C}$. A avaliação da superfície foi feita de acordo com a norma ASTM D-1666/87. O resultado mostrou que a madeira de Eucalyptus grandis apresenta um bom comportamento durante os testes de usinagem, obtendo notas 1 e 2 (excelente e bom), exceto para os testes de furação para a dobradiça no furo passante e furação com broca de 12,8 e $6 \mathrm{~mm}$, pois alcançaram notas entre 2 e 4 (bom, regular e ruim). O tratamento térmico diminuiu a quantidade de defeitos nas amostras usinadas, com exceção para o defeito grã arrancada, em algumas operações de usinagem.
\end{abstract}

Palavras-chave: usinagem da madeira; superfície da madeira; tratamento térmico.

\begin{abstract}
The objective of this study was to study the machined surface quality of Eucalyptus grandis wood submitted to heat treatment. The machining operations realized on the boards obtained from the processing of six trees were: planing, thicknessing, tear in horizontal drilling, drilling, drilling hinge and sanding. 208 boards of $125 \times 25 \times 500 \mathrm{~mm}$ were selected, with 104 untreated and 104 heat-treated boards at maximum temperature of $190^{\circ} \mathrm{C}$. The evaluation of the surface was performed according to ASTM D-1666/87 standard. The result showed that the Eucalyptus grandis wood presents a good behavior during the machining tests, obtaining notes 1 and 2 (excellent and good), except for drilling hinge test in the bolt hole and drilling with 12, 8, 6 $\mathrm{mm}$, reaching notes between 2 and 4 (good, regular and bad). The heat treatment reduced the number of defects on the machined samples, except for torn grain in some machining operations.
\end{abstract}

Keywords: wood machining; wood surface; heat treatment.

1 Engenheira Florestal, Dra., Professora Adjunta do Departamento de Produtos Florestais, Universidade Federal Rural do Rio de Janeiro, CEP 23890-000, Seropédica (RJ), gilpalermo@hotmail.com

2 Engenheiro Florestal, Dr., Professor Associado do Departamento de Produtos Florestais, Universidade Federal Rural do Rio de Janeiro, Rod. BR 465, Km 07, CEP 23890-000, Seropédica (RJ), Brasil. latorraca@ufrrj.br

3 Engenheiro Florestal, Dr., Professor Associado do Departamento de Produtos Florestais, Universidade Federal Rural do Rio de Janeiro, Rod. BR 465, Km 07, CEP 23890-000, Seropédica (RJ), Brasil. amcarvalho@ufrrj.br

4 Engenheira Florestal, Dra ., Professora Adjunto do Departamento de Produtos Florestais, Universidade Federal Rural do Rio de Janeiro, Rod. BR 465, Km 07, CEP 23890-000, Seropédica (RJ), Brasil. rosileigar@hotmail.com

Recebido para publicação em 30/03/2012 e aceito em 12/06/2013

Ci. Fl., v. 25, n. 1, jan.-mar., 2015 


\section{INTRODUÇÃO}

Usinagem da madeira é todo o processo pelo qual a forma de uma peça é modificada pela remoção progressiva de cavacos de tamanhos e formas variadas. Ela abrange tanto o processamento primário quanto o processamento secundário.

No processamento primário, as toras (peças cilíndricas) são transformadas em peças prismáticas (tábuas, pranchões, caibros, entre outros). No processamento secundário, as peças advindas do processamento primário são retrabalhadas através das operações de usinagem tais como o aplainamento, fresamento, furação, molduramento, lixamento e torneamento e ganham um melhor aspecto em suas superfícies.

$\mathrm{O}$ interesse daqueles que usinam a madeira é obter uma superfície de boa aparência e realizar, com qualidade, operações de acabamento com produtos de recobrimento, como tintas e vernizes. No entanto, mesmo após o aplainamento, o fresamento, a furação, o molduramento, o lixamento e o torneamento, irregularidades, como marcas e sulcos, ainda são aparentemente visíveis.

Essas irregularidades são geradas pela ferramenta de corte, as quais abrem cavidades nos diferentes tipos de células constituintes do tecido lenhoso. O tamanho dessas cavidades depende de diversos fatores que podem estar relacionados com a própria madeira (dureza, abrasividade, orientação da grã, resistência mecânica, etc.), com os parâmetros de corte (altura e profundidade de corte, espessura do cavaco, velocidade de corte e de alimentação, etc.) e ainda com o ângulo de ataque, ângulo de cunha, ângulo de incidência e afiação da ferramenta (FILHO, 2004).

Para compreender a influência desses fatores na qualidade da superfície, várias estratégias têm sido utilizadas, como exemplo, aquelas focalizadas na ferramenta, na direção da usinagem, na velocidade de alimentação e de corte e no tipo de tratamento dispensado à madeira (KORKUT e HIZIROGULU, 2008; KORKUT e GULLER, 2008; KORKUT et al., 2008; OLEK e BONARSKI, 2008; DE MOURA et al., 2008; LOPES, 2007; MADEIRA e CIA, 1995; VOVELLE e MELLOTTEE, 1982).

Dentre os tratamentos dispensados à madeira, vários estudos têm sido realizados sobre o efeito do tratamento térmico na qualidade da superfície usinada, visto que, quando a madeira é submetida a elevadas temperaturas $\left(150-250^{\circ} \mathrm{C}\right)$, a resistência ao corte diminui devido à degradação de seus componentes químicos fundamentais, sobretudo das hemiceluloses (KORKUT e HIZIROGULU, 2008; VOVELLE e MELLOTTEE, 1982).

Em consequência da redução da resistência do corte provocada pelo tratamento térmico, tábuas com melhor qualidade de superfície usinada podem ser produzidas em virtude do aumento da friabilidade da madeira, que permite a formação de um tipo de cavaco pequeno e mais quebradiço.

Neste contexto e considerando a viabilidade da utilização da madeira de Eucalyptus grandis na construção civil e na produção de móveis, este trabalho teve como objetivo avaliar a qualidade da superfície da madeira tratada termicamente através das operações de usinagem.

\section{MATERIAL E MÉTODO}

\section{Amostragem e preparo do material}

O material utilizado neste trabalho foi proveniente de seis árvores de Eucalyptus grandis Hill ex Maiden com vinte três anos de idade, cedido pela empresa QUINVALE, situada no município de Barra do Piraí - RJ. De cada árvore retiraramse três toras de $2,0 \mathrm{~m}$ de comprimento, que foram desdobradas em tábuas, com auxílio de uma serra de fita vertical para produção pranchas e uma serra circular para produção das tábuas.

As tábuas foram secas em estufa convencional até a umidade média final de $10 \%$ e classificadas segundo a norma ASTM D-1666 (1987). Após a classificação, com base nos estudos feitos pelo IBAMA (1997), as tábuas tiveram suas dimensões ajustadas para $125 \times 25 \times 500 \mathrm{~mm}$.

Foram selecionadas 208 amostras, sendo que destas, metade foi submetida ao tratamento térmico a uma temperatura final de $190^{\circ} \mathrm{C}$ por 3 horas, conforme metodologia descrita por Palermo (2010).

Em seguida, foram executados nas amostras tratadas e não tratadas termicamente, os testes de desempeno, desengrosso, lixamento, rasgo na furadeira horizontal, furação com broca de 12,8 e $6 \mathrm{~mm}$ e furação para dobradiça de acordo com os parâmetros descritos na Tabela 1 e norma ASTM D-1666 (1987).

Como o Eucalyptus grandis apresenta grã irregular, o desempeno foi feito nos dois sentidos da amostra de madeira, sendo metade da amostra desempenada a favor da grã e a outra metade contra a grã (Figura 1). 
TABELA 1: Parâmetros utilizados nos testes de usinagem, por operação.

TABLE 1: Parameters used in the machining tests by operation.

\begin{tabular}{lcccccc}
\hline \multicolumn{1}{c}{ Operação } & $\mathrm{Z}\left(\mathrm{n}^{\circ}\right)$ & $\mathrm{RPM} \mathrm{n}\left(\mathrm{min}^{-1}\right)$ & $\mathrm{D}(\mathrm{mm})$ & $\mathrm{V}_{\mathrm{c}}(\mathrm{m} / \mathrm{s})$ & $\mathrm{Vf}(\mathrm{m} / \mathrm{min})$ & $\mathrm{Fz}$ \\
\hline Desempeno & 2 & 3400 & 100 & 18,0 & 15 & 2,2 \\
Desengrosso & 2 & 2600 & 120 & 17,0 & 8 & 1,5 \\
Rasgo furadeira horizontal & 2 & 3500 & 8 & 1,4 & Manual & - \\
Furação normal (6, 8 e 12) & 2 & 3100 & $6 ; 8 \mathrm{e} 12$ & 0,$9 ; 1,2 \mathrm{e} 1,95$ & Manual & - \\
Furação dobradiça & 2 & 3100 & 26 & 4,2 & Manual & - \\
Lixamento & - & 600 & 250 & 7,8 & & \\
\hline
\end{tabular}

Em que: $Z$ = Número de gumes da ferramenta de corte, RPM = frequência de rotação do eixo porta ferramenta, $\mathrm{D}=$ Diâmetro da ferramenta, $\mathrm{Vc}=$ velocidade de corte, $V f=$ velocidade de avanço e $f_{z}=$ avanço por dente.

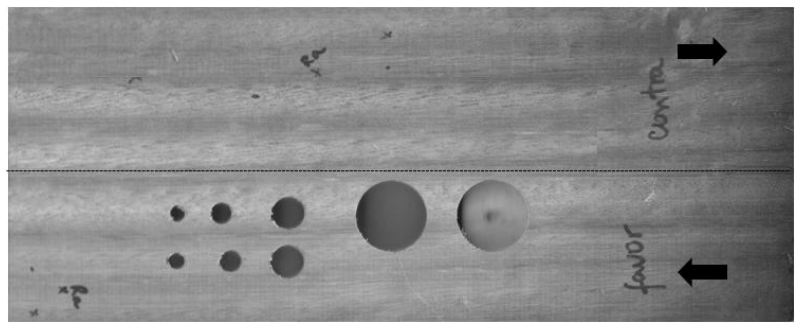

FIGURA 1: Sentidos da operação de desempeno. FIGURE 1: Direction of the planing operation.

\section{Avaliação das operações de usinagem}

A avaliação das superfícies usinadas, de todos os testes realizados, foi feita de forma subjetiva, com base na norma ASTM D-1666/87 e nos estudos de Goli et al. (1997). O sistema de classificação empregado para a avaliação qualitativa da superfície usinada encontra-se na Tabela 2.

As peças que receberam notas 1 e 2 foram consideradas aprovadas para serem utilizadas em produtos cuja qualidade da superfície é primordial, tais como, móveis, pisos, portas, janelas, batentes, entre outros. As peças que receberem notas de 3 a 5 foram consideradas aprovadas para serem utilizadas em produtos que não exigem uma textura muito fina, como por exemplo, caibros, tábuas e portões
(CASTRO SILVA, 2002; SILVA, et al., 2009; SOUZA, 2007).

\section{RESULTADOS E DISCUSSÃO}

\section{Avaliação das operações de usinagem}

Durante a avaliação da superfície usinada, os defeitos que mais ocorreram foram grã arrancada, grã felpuda ou lanosa, levantamento de fibras e grã comprimida. Como os defeitos não foram semelhantes para todas as operações, optou-se por avaliar cada uma delas separadamente.

\section{Desempeno}

A Figura 2 mostra a tendência de variação dos defeitos ocorridos na madeira tratada e não tratada termicamente, durante a operação de desempeno na direção da grã e contra a grã.

Observa-se que a madeira que recebeu o tratamento térmico obteve os melhores resultados, ainda que a madeira não tratada termicamente de Eucalyptus grandis tenha apresentado um bom desempenho frente a essa operação.

Quanto à direção de usinagem, observa-se

TABELA 2: Sistema de avaliação da qualidade da superfície (ASTM D-1666/87).

TABLE 2: Evaluation system of the surface quality (ASTM D-1666/87).

\begin{tabular}{ccc}
\hline Nota & Grau & Significado \\
\hline 1 & Ausência de defeitos & Excelente \\
2 & Superfície da peça usinada afetada em menos de $50 \%$ pelo defeito & Bom \\
3 & Superfície da peça usinada afetada em $50 \%$ pelo defeito & Regular \\
4 & Superfície da peça usinada afetada em mais de $50 \%$ pelo defeito & Ruim \\
5 & Superfície da peça usinada afetada em $100 \%$ pelo defeito & Muito ruim \\
\hline
\end{tabular}


que a qualidade da superfície da peça na operação de desempeno foi favorecida quando esta foi realizada a favor da grã (FG).

O defeito arrancamento das fibras foi maior na madeira tratada termicamente na operação de desempeno contrário às fibras.

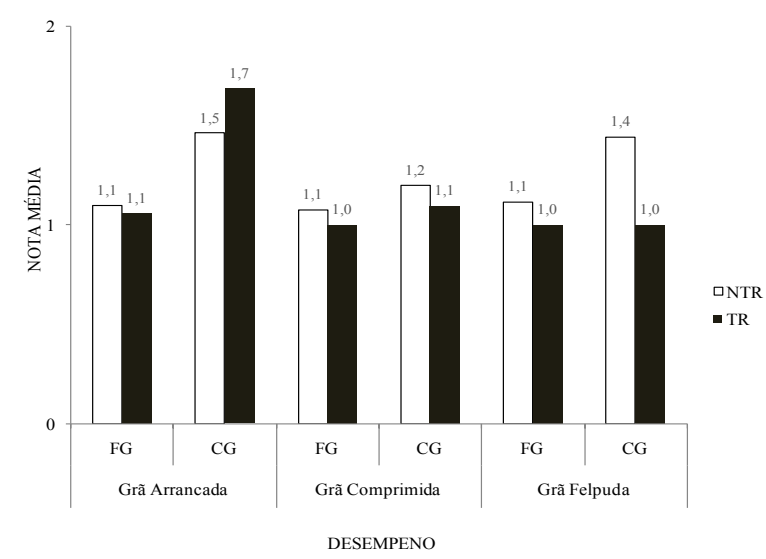

FIGURA 2: Notas médias atribuídas aos defeitos ocorridos durante a operação de desempeno da madeira não tratada termicamente (NTR) e tratada termicamente (TR), a favor da grã (FG) e contra a grã (CG).

FIGURE 2: Average notes attributed to defects occurring during planing tests of untreated (NTR) and heat treated (TR) woods on direction of the fibers (FG) and against the fibers $(\mathrm{CG})$.

Confrontando os resultados encontrados neste estudo com os de Castro Silva (2002) e Silva (2002) para Eucalyptus grandis e Souza (2007) para Eucalyptus camaldulensis e Eucalyptus urophylla observa-se que em geral, as notas alcançadas pelas superfícies aplainadas foram inferiores.

Castro Silva (2002) verificou que a madeira de Eucalyptus grandis apresentou um bom desempenho no teste de aplainamento $(88,3$ a $68,3 \%$ de aprovação) e a porcentagem de peças aprovadas aumentou nas árvores com maiores idades.

Souza (2007), durante teste de aplainamento da madeira de clones de eucalipto, verificou que $100 \%$ das amostras obtiveram notas 1 e 2 , o que indica um bom desempenho da madeira ao teste e boa adequação do maquinário e ferramentas. Em relação aos defeitos observados verificouse uma maior frequência de queima da superfície e arrancamento de fibras e menor frequência de arrepiamento de fibras e aspereza da superfície.

Quanto ao efeito do tratamento térmico, resultados similares foram observados por De Moura et al. (2008), os quais observaram uma redução da ocorrência de defeitos nas superfícies aplainadas das madeiras de eucaliptos e pinus tratadas termicamente. Segundo esses autores, quanto maior a temperatura de tratamento maior é a qualidade das superfícies aplainadas.

\section{Desengrosso}

A Figura 3 mostra a tendência de variação dos defeitos ocorridos na superfície das peças de madeira tratada e não tratada termicamente submetidas ao desengrosso. Observa-se que a madeira de Eucalyptus grandis apresentou um bom desempenho frente a essa operação, pois a média geral das notas atribuídas para os defeitos variou de 1,5 a 2,2 .

Com relação ao tipo de tratamento aplicado à madeira, observa-se que a madeira tratada apresentou menor ocorrência do defeito grã felpuda e maior incidência de grã arrancada. A maior ocorrência de grã arrancada pode ser atribuída à maior fragilidade da madeira frente à ação da ferramenta cortante, visto que o tratamento térmico deixa a madeira mais seca e diminui sua densidade.

Confrontando as notas obtidas neste trabalho, para os defeitos grã arrancada e grã felpuda da madeira não tratada, com os resultados encontrados por Castro Silva (2002) e Silva (2002) para Eucalyptus grandis verifica-se que são similares, pois ambos estão entre as classes 1 e 2 (excelente e bom).

\section{Rasgo na furadeira horizontal}

A Figura 4 mostra a tendência de variação dos defeitos ocorridos na superfície das peças de madeira tratada e não tratada termicamente durante o teste de rasgo. Observa-se que as notas recebidas variaram de 1,1 a 2,0, permitindo classificar as madeiras tratadas e não tratadas termicamente nas classes excelente e bom.

Com relação ao tratamento, verifica-se que o mesmo causou uma redução na quantidade de defeitos de grã arrancada (Figura 4a) e grã levantada (Figura 4b).

Para esta operação o resultado obtido é semelhante ao encontrado por Castro Silva (2002), que verificou que quase $100 \%$ das amostras de 


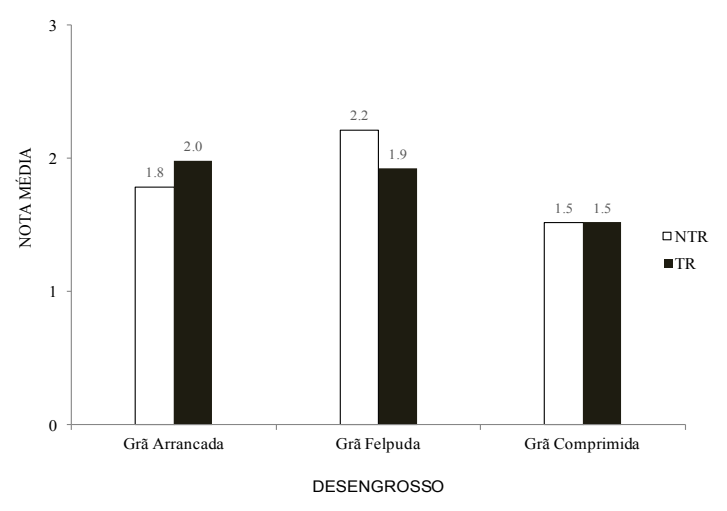

FIGURA 3: Notas médias atribuídas aos defeitos ocorridos durante a operação de desengrosso da madeira não tratada (NTR) e tratada (TR) termicamente.

FIGURE 3: Average notes attributed to defects occurring during thicknessing tests of untreated (NTR) and heat-treated (TR) woods.

Eucalyptus grandis com quatorze, vinte e vinte cinco anos de idade foram classificadas dentro da classe excelente. Apenas as amostras de eucalipto de 10 anos obtiveram notas 2 e 3 (bom e regular). Souza (2007) também verificou que madeiras de clones de eucalipto apresentaram um bom desempenho, visto que todas as peças foram aprovadas, obtendo notas 1 e 2 .

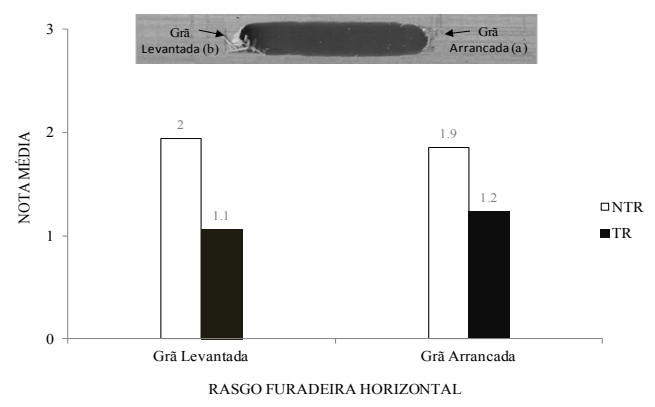

FIGURA 4: Notas médias atribuídas aos defeitos ocorridos durante a operação de rasgo da madeira não tratada (NTR) e tratada (TR) termicamente.

FIGURE 4: Average notes attributed to defects occurring during tear in horizontal drilling tests of untreated (NTR) and heat-treated (TR) woods.

\section{Furação com broca de 12, 8 e 6 mm}

Na Figura 5 observa-se que a média geral das notas alcançadas pelas amostras de madeira variou de 1,3 a 3,6 permitindo enquadrar a madeira de eucalipto nas classes bom e regular. Para o defeito grã arrancada (5a), a madeira tratada termicamente apresentou o pior desempenho, pois obteve nota média entre 2,6 e 3,6.

As notas obtidas neste estudo para a madeira não tratada foram superiores às encontradas por Castro Silva (2002) para Eucalyptus grandis, por Souza (2007) e Carvalho (2006) para Eucalyptus urophylla. Souza (2007), ao estudar o comportamento da madeira de Eucalyptus urophylla durante o teste de furação, verificou que $96 \%$ das amostras de madeira de árvores com 29 anos de idade foram enquadradas na classe 1 (excelente). Em madeira, oriunda de árvores com 10 e 8 anos, $64 \%$ e $53 \%$ das amostras foram enquadradas nas classes 2 (bom) e 3 (regular), respectivamente. Carvalho (2006) também verificou que $80 \%$ das amostras de Eucalyptus urophylla receberam notas 2 (bom) e 3 (regular).

Segundo Castilho e Cueto (1996), a qualidade da superfície furada depende do tipo de madeira, da ponta da broca, da densidade e da velocidade de avanço da broca. Segundo os autores, as madeiras de maior densidade tendem a apresentar menos defeitos de grã arrancada e grã levantada durante a operação de furação. No entanto, existem algumas madeiras que fogem à regra, pois elas possuem alto conteúdo de extrativos e sílica que interferem no fio da ferramenta de corte, gerando defeitos de usinagem.

\section{Furação para dobradiça}

A Figura 6 mostra a tendência de variação dos defeitos ocorridos na superfície das peças de madeira tratada e não tratada termicamente durante o teste de furação para dobradiça, no furo passante (P) e não passante (NP).

A nota alcançada pelas amostras de madeira para os defeitos grã felpuda e grã arrancada permite enquadrá-los nas classes excelente e bom. No entanto, para a grã arrancada, tanto a madeira tratada, quanto a madeira não tratada apresentaram desempenho insatisfatório, sendo enquadrada na classe ruim. A obtenção de superfícies de baixa qualidade foi devido à utilização de altas velocidades de avanço e retirada brusca da broca no término da 


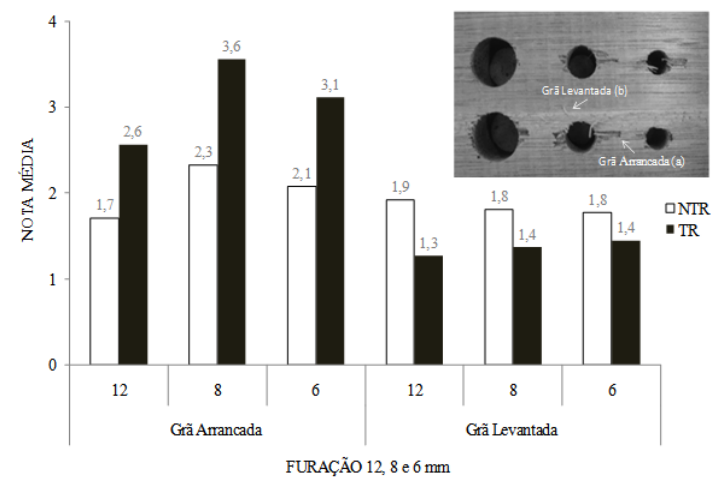

FIGURA 5: Notas médias atribuídas aos defeitos ocorridos durante a operação de furação com broca de 12,8 e $6 \mathrm{~mm}$ da madeira não tratada (NTR) e tratada (TR) termicamente.

FIGURE 5: Average notes attributed to defects occurring during drilling tests with drill of 12,8 and $6 \mathrm{~mm}$ of untreated (NTR) and heat-treated (TR) woods.

furação.

Com relação ao tratamento, verifica-se que o mesmo reduziu a ocorrência de defeitos na madeira, exceto para o defeito grã arrancada (Figura 6a) no furo passante. Observa-se ainda que para os defeitos grã felpuda (Figura 6b) e grã levantada, as amostras de madeira tratada termicamente apresentaram um excelente comportamento, pois receberam nota 1 .

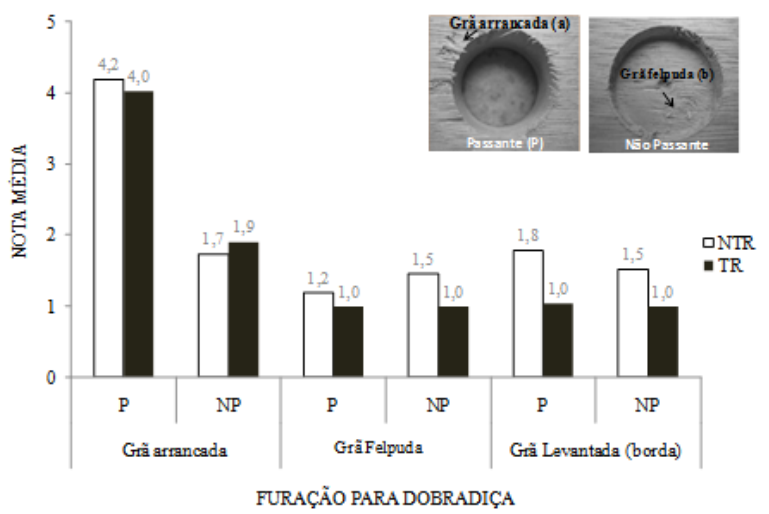

FIGURA 6: Notas médias atribuídas aos defeitos ocorridos durante a operação de furação para dobradiça da madeira não tratada (NTR) e tratada (TR) termicamente.

FIGURE 6: Average notes attributed to defects occurring during drilling hinge tests of untreated (NTR) and heat-treated (TR) woods.

\section{Lixamento}

As Figuras 7 ( $a$ e b) mostram a tendência de variação dos defeitos ocorridos nas peças de madeira tratada e não tratada termicamente, lixadas com lixas de granulometria 80 e 100 . O lixamento foi efetuado sobre a superfície que havia sido anteriormente desempenada e desengrossada.

Para o lixamento com lixa de granulometria 80 (Figura 7a), observa-se que a média geral das notas dos defeitos grã felpuda e comprimida variou entre 1,1 e 1,9. Este resultado confere à madeira de Eucalyptus grandis conceito excelente e bom frente a esta operação.

Para o lixamento com lixa de granulometria 100 observa-se na média geral das notas foram inferiores aos valores obtidos para a lixa de granulometria de 80 (Figura 7b).

Verifica-se ainda que o tratamento térmico melhorou a qualidade das superfícies lixadas, exceto para o defeito grã comprimida, com lixa de granulometria 100, no qual os valores são semelhantes.

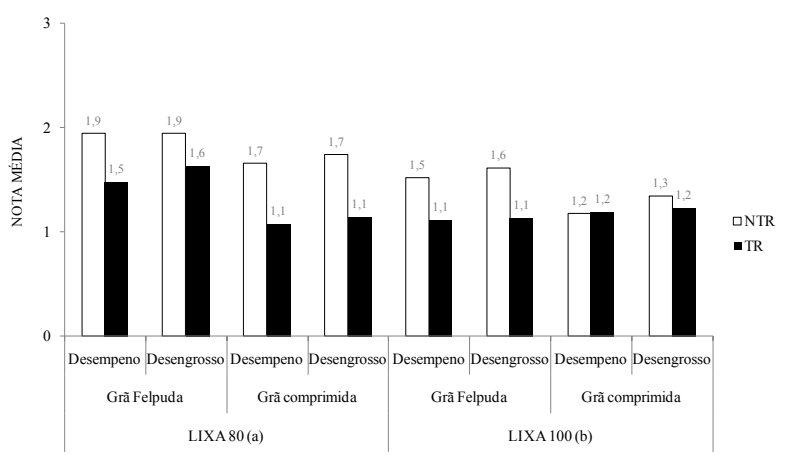

FIGURA 7: Notas médias atribuídas aos defeitos ocorridos durante a operação de lixamento, com lixa de granulometria 80 (a) e 100 (b), da madeira não tratada (NTR) e tratada (TR) termicamente.

FIGURE 7: Average notes attributed to defects occurring during sanding tests with sandpaper sizes of 80(a) and 100 (b) of untreated (NTR) and heat-treated (TR) woods.

Informações sobre as superfícies lixadas de eucaliptos são escassas. No entanto, comparando os resultados deste trabalho com os obtidos por Malkoçoglu e Özdemir (2007) para a espécie de Fagus orientalis, Castanea sativa, Alnus glitunosa, Pinus sylvestris e Picea orientalis, verifica-se que o Eucalyptus grandis apresentou alto desempenho 
durante o teste, pois recebeu nota 1 (excelente) e 2 (bom).

A diminuição da ocorrência de defeitos na madeira após o lixamento com lixa de granulometria 100 está de acordo com resultados obtidos por Hall e Heard (1982), Lihra e Ganev (1999) citado por De Moura (2006), que destacam que as irregularidades deixadas por lixas mais grosseiras diminuem quando a madeira é submetida a uma nova operação de lixamento com lixa de granulometria mais fina.

Segundo Taylor et al. (1999), as características da madeira, a pressão exercida, o tipo de mineral abrasivo, a orientação do movimento da lixa e a dimensão dos grãos abrasivos são os parâmetros mais importantes do processo de lixamento.

\section{CONCLUSÕES}

Com base nos resultados dos testes de usinagem conclui-se que:

As superfícies das amostras submetidas ao teste de desempeno, desengrosso, rasgo na furadeira horizontal, lixamento com lixa de granulometria 80 e 100 obtiveram notas 1 e 2 (excelente e bom), podendo ser utilizadas na confecção de Produtos de Maior Valor Agregado (PMVA);

As amostras desempenadas a favor da grã apresentaram menos defeitos;

$\mathrm{Na}$ furação para a dobradiça no furo passante e na furação com broca de 12,8 e $6 \mathrm{~mm}$, a madeira apresentou para o defeito grã arrancada, comportamento de regular a ruim;

Em geral o tratamento térmico melhorou a qualidade das superfícies usinadas.

\section{REFERÊNCIAS BIBLIOGRAFICAS}

AMERICAN SOCIETY FOR TESTING AND MATERIALS. ASTM D 1666-87 standard method for conducting machining tests of wood and wood base materials (reapproved 1994). Philaldelphia, p. $226-245,1995$.

CASTILHO, J. L. M.; CUETO, E. M. Características de maquinado de 32 especies de madera. Madera y Bosques v. 2, n. 1, p. 45-46, 1996.

CASTRO SILVA, J. C. Caracterização da madeira de Eucalyptus grandis Hill ex Maiden, de diferentes idades, visando a sua utilização na indústria moveleira. 2002. $160 \mathrm{f}$. Tese (Doutorado em Ciência Florestal) - Universidade Federal do Paraná - UFPR, Curitiba, 2002.
CARVALHO, D. S. Avaliação da secagem ao ar livre e usinagem da madeira de Eucalyptus urophylla para produção de móveis. 2006. 49 f. Monografia (Curso de Engenharia Florestal) Universidade Federal Rural do Rio de Janeiro, Seropédica, 2006.

DE MOURA, L. F. Étude de trois procédés de finition des surfaces du bois d'érable à sucre pour fins de vernissage. 2006. 158 f. Tese (Doutorado) Faculté de Foresterie et de Géomatique, Université Laval, Québec, 2006.

DE MOURA, L. F. et al. Efeito da termorretificação sobre as propriedades de usinagem de Eucalyptus grandis e Pinus caribae var. hondurensis. In: I SIMPÓSIO DE CIÊNCIA E TECNOLOGIA DA MADEIRA DO ESTADO DO RIO DE JANEIRO, Anais..., UFRRJ/RJ. Dez. 2008.

FILHO, F. C. L. Análise da usinagem da madeira visando a melhoria de processos em indústrias de móveis. 2004. 176 f. Tese (Doutorado em Engenharia de Produção) - Universidade Federal de Santa Catarina, Florianópolis, 2004.

GOLI, G. et al. Classificazione qualitativa dei difetti con metodo visuale. Xylon, p. 44-48, marzo 2004.

GOLI, G. et al. Industrial machining of Douglas fir with various tools and materials. In: IWMS, 15. 2001, Los Angeles. Proceedings..., Los Angeles (USA), july/aug., 2001, p. 173.

GOLI, G. et al. The machining process of the european Douglas fir: The surface quality. In: Proceedings..., Belgrado (Serbia), vol. II, Set/oct., 1997, p. 473.

HALL, A.; HEARD, J. Wood finishing: Furniture finishing. $1^{\text {st }}$ ed. New York: Holt, Rinehart and Winston, 1982, $196 \mathrm{p}$.

IBAMA MARQUES, M. H. B. et al. Madeiras da Amazônia: Características e Utilização. IBAMA/ DIRPED/LPF, 1997, $141 \mathrm{p}$.

KORKUT, D. S.; GULLER, B. The effects of heat treatment on physical properties and surface roughness of red-bud maple (Acer trautvetteri Medw.) wood. Bioresource Technology, v. 99, p. 2846-2851, 2008.

KORKUT, S.; HIZIROGLU, S. Effect of heat treatment on mechanical properties of hazelnut wood (Corylus colurna L.). Materials and Design, p. 1-6, 2008.

KORKUT, S. et al. The effects of heat treatment on technological properties in Red-bud maple (Acer trautvetteri Medw.) wood. Bioresource Technology, v. 99, p. 1538-1543, 2008.

LOPES, C. S. D. Caracterização da madeira de 
três espécies de eucalipto para uso em movelaria. 2007. 88 f. Dissertação (Mestrado em Recursos Florestais) - ESALQ- USP, Piracicaba, São Paulo, 2007.

MADEIRA \& CIA. A madeira como matéria-prima. Madeira \& Cia, v. 4, n. 19, p.18-21, 1995.

MALKOÇOĞLU,A.; ÖZDEMIR, T. The machining properties of some hardwoods and softwoods naturally grown in Eastern Black Sea Region of Turkey. Building and Environment, v. 42, n. 7, p. 2562-2567, 2007.

MONTERO, J. R. S.; LIZANO, R. E. R. Ensayos de trabajabilidad de Tectona grandis L. f. de 11 años de edad proveniente de dos sitios de crescimiento en plantaciones del Atlántico y el pacifico de Costa Rica. Kurú: Revista Florestal, v. 5, n. 14, p. 1-14, 2008.

NEGRI M.; GOLI G. Qualità delle superfici lavorate del legno di Abete rosso e di Douglasia valutata con una opportuna classificazione visuale. Legno Cellulosa Carta, v. 6, n. 1, p.10-21, 2001.

OLEK, W.; BONARSKI, J. T. Texture changes in thermally modified Wood. Scopus. Archives of Metallurgy and Materials, v. 53, n. 1, p.207-211, 2008.

PALERMO, G. P. M. Propriedades e comportamento tecnológico da madeira de Eucalyptus grandis W. Hill Ex-Maiden visando a sua utilização em produtos de maior valor agregado. 2010. 238f. Tese (Doutorado em Ciências Ambientais e Florestais) - Universidade Federal
Rural do Rio de Janeiro, Seropédica, 2010.

PETROCCHI, S. Prove di lavorabilità e di abrasione degli utensili su alcune specie legnose. 1987. Tesi (di láurea) - Università degli studi di Firense, Facoltà di Agraria, corso di laurea in Scienze Forestali, 1987.

SILVA, J. R. M. et al. Parâmetros de qualidade da usinagem para determinação dos diferentes usos da madeira de Eucalyptus. Cerne, v. 15, n. 1, p. 75-83, jan./mar. 2009.

SILVA, J. R. M. 2002. Relações da usinabilidade e aderência do verniz com as propriedades fundamentais do Eucalyptus grandis Hill ex. Maiden. 2002. 179 f. Tese (Doutorado em Ciências Florestais), Setor de Ciências Agrárias da Universidade Federal do Paraná, Curitiba, 2002.

SOUZA, M. O. A. Madeira de Eucalyptus camaldulensis Dehnh e Eucalyptus urophylla S. T. Blake visando seu uso na indústria moveleira. 2007. 91p. Dissertação (Mestrado em Ciência Florestal) - Universidade Federal de Viçosa, Viçosa, 2007.

TAYLOR, J. B. et al. Quantification of process parameters in wood sanding operation. Forest Products Journal, v. 49, n. 5, p. 41-46, 1999.

VOVELLE, C.; MELLOTTEE, H. Energy from biomass. Modelisation de la pyrolyse oxydante ou noxydante de bois ou de déchéts végetaux à partir de leurs composants. In: PALZ, W.; CHARTIER, P. (Eds.), 2. ed. London: Applied Sciences, 1982, p. 925-929. 